RBMC

ISSN 2447-9071

doi https://doi.org/10.36414/rbmc.v7i19.112
Contato para correspondência: Anna Caroliny Souza Silva

E-mail:

anna.silva@faculdadepm.edu.br

Conflito de interesse: Não

Financiamento: Recursos próprios

Recebido: 19/11/2021

Aprovado: 15/12/2021

\section{Prevalência do uso de drogas de abuso por policiais militares do estado de Goiás no período de 2016 a 2021}

\section{Prevalence of drug use of abuse by military police of the state of Goiás in the period from 2016 to 2021}

Grazielli Lisboa da Silva Schimidt ${ }^{1}$, Anna Caroliny Souza Silva ${ }^{1}$, Jessica Cristina Santos de Oliveira ${ }^{1}$, Vania Cristina Rodriguez Salazar ${ }^{1,2}$

${ }^{1}$ Faculdade da Polícia Militar - FPM

${ }^{2}$ Pontifícia Universidade Católica de Goiás - PUC Goiás

\section{Resumo}

Ouso de drogas lícitas e ilícitas écertamente um problema de cunho social. Existem inúmeros motivos pelos quais uma pessoa começa a fazer uso dessas substâncias, dentre elas: frustrações, curiosidade, experiências agradáveis, "criativas", "viajar", escapar de problemas, entre outros. Normalmente esse contato inicial com as drogas ocorre pela dificuldade de enfrentar as diferentes situações do cotidiano. Nesse contexto, diversas classes profissionais que vivenciam um cotidiano estressante, enveredam por este caminho, dentre elas, os policiais militares. Dessa forma, o objetivo deste artigo foi realizar um levantamento dos resultados de exames toxicológicos obtidos em um hospital de atendimento ao policial militar do Estado de Goiás no período de 2016 a 2021. Esse levantamento foi realizado através de análise toxicológica em 632 amostras de urina, submetidas a testes imunocromatográficos qualitativos. Foram pesquisadas 12 drogas e/ou metabólitos, das quais:anfetaminas, metanfetaminas, cocaína, tetrahidrocanabinol, benzodiazepinas, morfina, metilenodioximetanfetamina, propoxifeno, antidepressivos tricíclicos, fenciclidina, barbitúricos emetadona. Os resultados obtidos foram: das 632 amostras analisadas, a maioria delas (628) foram de policiais do gênero masculino com faixa etária entre 20 e 35, a prevalência de drogas foi de 28 amostras positivas (4,4\%), sendo que as drogas de abuso mais encontradas foram: benzodiazepínicos, maconha, cocaína, opiáceos e antidepressivos havendo associação no uso dessas drogas em alguns casos. Esses dados mostram a necessidade de políticas específicas de controle e prevenção ao consumo de drogas.

Palavras-Chave: Drogas de Abuso; Urina; Policiais militares; Imunocromatografia.

\begin{abstract}
The use of licit and illicit drugs is certainly a social problem. There are numerous reasons why a person begins to use these substances, among them: frustrations, curiosity, pleasant, "creative" experiences, "travel", escape from trouble, among others. Usually, this initial contact with drugs occurs due to the difficulty of facing the different everyday situations. In this context, several professional classes that experience astressful daily life, go down this path, among them, the military police. Thus, the aim of this article was to perform a survey of the results of toxicological tests obtained in a hospital for the care of the military police officer of the State of Goiás in the period from 2016 to 2021. This survey was performed through toxicological analysis in 632 urine samples submitted to qualitative immunochromatographic tests. Twenty drugs and/ormetabolites were surveyed:amphetamines, methamphetamines, cocaine, tetrahydrocannabinol, benzodiazepines, morphine, methylenedioxymethamphetamine, propoxifene, tricyclic antidepressants, phencycldine, barbiturates and methadone. The results obtained were: of the 632 samples analyzed, most of them (628) were male police officers aged between 20 and 35, the prevalence of drugs was 28 positive samples (4.4\%), and the most common abuse drugs were: benzodiazepines, marijuana, cocaine, opiates and antidepressants, and there was an association in the use of these drugs in some cases. It was concluded that there was an increase in the use of licit psychoactive substances by the police class, thus requiring specific policies for control and prevention of drug use. Keywords: Drug Abuse; Urine; Military police; Immunochromatography.
\end{abstract}




\section{Introdução}

A prática do uso abusivo de psicotrópicos trata-se de um grave problema de saúde pública'. Para a Organização Mundial de Saúde (OMS), substâncias que agem no Sistema Nervoso Central (SNC) e produzem alterações comportamentais, humorais e cognitivas são consideradas psicotrópicas ou psicoativas $^{2}$. De acordo com o efeito que as substâncias psicotrópicas provocam no SNC, elas dividem-se em três diferentes grupos, podendo ser depressoras, estimulantes e perturbadoras ${ }^{3}$.

Segundo a OMS, independentemente de fatores sociais como idade, sexo e nível de instrução, cerca de $10 \%$ da população no mundo consomem este tipo de substância de forma abusiva, seja como drogas ilícitas ou lícitas ${ }^{4}$. Dentre as substâncias psicoativas lícitas estão os medicamentos utilizados no combate à: ansiedade, depressão, angústia, insônia e agitação ${ }^{5}$. O consumo exacerbado dessas substâncias é uma preocupação de âmbito mundial, uma vez que causa grande impacto social e econômico ${ }^{6}$.

De acordo com o Relatório Mundial sobre Drogas, em 2012, $1 \%$ dos óbitos de adultos são causados pelo consumo de drogas ilícitas. Dentre as drogas ilícitas mais utilizadas no mundo está a Cannabis (com uma taxa de prevalência anual de 2,6\% a 5,0\%) e estimulantes do tipo anfetamina (excluindo ecstasy), com taxa de $0,3 \%$ a 1,2\%. Com relação às drogas lícitas, na população adulta mundial, a prevalência do consumo de tabaco é de $25 \%$ e a de álcool é de $42 \%$, ambas as drogas apresentam uma prevalência cinco e oito vezes maior do que o uso de drogas ilícitas (com 5,0\% de prevalência), respectivamente 7 .

Os dados de um levantamento mais recente do Relatório Mundial sobre Drogas, em 2020, mostram que cerca de 269 milhões de pessoas usaram drogas no mundo em 2018 com um aumento de 30\% em comparação a 20098. Com relação ao consumo de drogas no Brasil, a Fundação Oswaldo Cruz (Fiocruz) e parceiros, realizaram, em 2017, o $3^{\circ}$ Levantamento Nacional sobre o Uso de Drogas pela População Brasileira, compreendendo as capitais de todas as regiões do país (Norte, Nordeste, Sudeste, Sul, Centro-Oeste), onde estimou-se a prevalência de: 1. Pessoas que fazem uso de maconha regularmente, 3,1\%; 2. Pessoas que somente fazem uso de substâncias ilícitas, sendo: haxixe ou skank (derivados da planta Cannabis), cocaína em pó, crack e similares (merla, pasta base e oxi), solventes, ecstasy, ayahuasca, dietilamida do ácido lisérgico (LSD), quetamina e heroína 1,9\%; 3. Pessoas que somente fazem uso de crack e/ou similares 1,1\% ${ }^{9}$. Esses dados impactam de forma significativa em vários aspectos sociais, como saúde e segurança pública.

Sabe-se que algumas populações estão mais sujeitas a essa prática, como por exemplo, pessoas em situação de rua que, devido à sua vulnerabilidade, geralmente acabam fazendo uso abusivo de álcool e drogas ilícitas. Outros fatores como, ocorrência de desordem mental, círculo familiar enfraquecido e falta de escolaridade podem acabar aumentando essa reincidência ao uso ${ }^{10}$. A classe adolescente é uma das classes que mais está sujeita ao uso de drogas, por diversas motivações, como busca por diversão, curiosidade, necessidade de pertencer a um determinado grupo social, alívio do tédio, relaxamento e problemas pessoais ${ }^{11}$.

Outra classe da população que também é muito atingida pelo uso de substâncias psicoativas (SPA), é a dos profissionais da saúde, dentre eles, os enfermeiros. Acerca dos fatores desencadeadores, encontram-se as condições de trabalho em situações de extremo estresse ${ }^{12}$. Além disso, 70,5\% dos enfermeiros entrevistados em um estudo efetuado num Hospital da cidade do Paraná fizeram uso de psicofármacos e desse percentual, $44 \%$ se automedicavam com a justificativa de ter fácil acesso ao medicamento ${ }^{13}$. Em relação a esse fácil acesso à droga, Bahora et al., realizaram um estudo em Atlanta, no período de 2002 a 2007, onde foi relatado por um dos 112 entrevistados, usuários de ecstasy, que o acesso para obtenção desses medicamentos é de tamanha facilidade, comparando a compra com um pacote de batatas fritas ${ }^{14}$. Há de se apontar, ainda, a classe policial que, também, é bastante acometida e como qualquer organização civil, não está isenta das adversidades relacionadas à prática do uso abusivo de substâncias psicoativas ${ }^{15,16}$.

No Brasil, a consequência do uso de substâncias psicotrópicas lícitas e ilícitas nos indicadores de mortalidade e morbidade da população é muito relevante. Isso apesar de que o número de registros de mortes causadas pelo uso abusivo da maioria dessas substâncias seja ainda muito pequeno, pois geralmente não se correlacionam patologias próprias do consumo excessivo de drogas como causa de morte. Em 2007, a taxa de mortalidade brasileira relacionada ao uso de drogas foi de 4,3 por 100.000 habitantes ( $90 \%$ das mortes foram relacionadas ao alcoolismo), dita taxa foi maior do que alguns países como Argentina 1,9; Chile 2,3; Estados Unidos 2,4, e menor do que outros países como França 5,6; Alemanha 6,2; Rússia 5,8 ${ }^{17}$. Ainda assim, são relativamente poucos os estudos mostrando a realidade do uso de substâncias psicotrópicas no Brasil, principalmente, no que tange às profissões, sobretudo, direcionados à classe policial.

Dessa forma, o objetivo deste artigo foi realizar um levantamento dos resultados de exames toxicológicos obtidos em um hospital de atendimento ao policial militar do Estado de Goiás no período de junho de 2016 a janeiro de 2021.

\section{Métodos}

\section{Tipo de Estudo}

Trata-se de um estudo transversal, no qual foi realizado um levantamento de dados secundários, tais como gênero, idade e 
resultado da análise toxicológica de amostras de urina do banco de dados do laboratório do Policial Militar do Estado de Goiás.

\section{Considerações Éticas}

Este trabalho foi aprovado pelo Comitê de Ética em pesquisa do hospital e maternidade Dona Íris com número de parecer 3.823.710 e CAAE 25742719.1.0000.8058.

\section{Análise Estatística}

A análise estatística foi realizada no programa Microsoft Office Excel ${ }^{\circledR}$, versão 2010.

\section{População estudada}

Foram analisadas um total de 632 amostras de urina de militares da ativa, integrantes do quadro de servidores da polícia militar do Estado de Goiás, no período de junho de 2016 a janeiro de 2021.

\section{Análise de urina}

As amostras de urina foram coletadas em recipientes limpos e secos, de plástico ou vidro, sem conservantes. Refrigeradas entre $2^{\circ} \mathrm{C} \mathrm{e} 8^{\circ} \mathrm{C}$ e armazenadas por até 48 horas. Amostras com precipitado visível foram filtradas, centrifugadas ou colocadas em repouso para a obtenção de um sobrenadante límpido.

Para a análise toxicológica da urina, foi utilizado o teste rápido de triagem para identificação de drogas de abuso, denominado Multi Drogas Rapid Test (12 drogas) marca ABON ${ }^{\circledR}$.

Vale ressaltar que, embora o teste seja muito preciso, existe possibilidade de resultados falsos devido à presença de substâncias interferentes na urina. As amostras caracterizadas como positivas não foram enviadas para análise confirmatória, pois a realização das mesmas, foi em razão de se obter informações para serem usadas pela junta médica da corporação. Entretanto, todas as amostras positivas foram devidamente armazenadas, caso fosse solicitado para confirmações médicas ou pedidos judiciais.

\section{Resultados}

Os dados das 632 amostras biológicas de urina foram tabulados e analisados para obtenção de dados sociodemográficos e de prevalência do uso de drogas de abuso por policiais militares do Estado de Goiás.

O primeiro parâmetro avaliado nas amostras foi o gênero, sendo que das 632 amostras analisadas no período estudado, $628(99,4 \%)$ foram provenientes de policiais do gênero masculino e $4(0,6 \%)$ do gênero feminino.

A segunda variável estudada foi a idade dos policiais, estratificada em três grupos: 20-25 anos, 26-34 anos e >35 anos. Dentre as 3 faixas etárias analisadas, a maior foi de 26-34 anos, com 376 amostras do gênero masculino e 3 amostras do gênero feminino, totalizando 379 amostras, nessa faixa etária.

A tabela 1 mostra os dados obtidos referentes ao gênero e à idade dos policiais militares que fizeram parte do estudo.

Tabela 1. Idade e gênero de policiais militares que participaram da pesquisa sobre drogas de abuso no Estado de Goiás durante o período de junho de 2016 a janeiro de 2021. ( $\mathrm{N}$ = número de policiais. $\%=$ porcentagem de policiais em cada faixa etária).

\begin{tabular}{ccccccc}
\hline & \multicolumn{3}{c}{ Gênero } \\
Faixas etárias & \multicolumn{2}{c}{ Masculino } & \multicolumn{2}{c}{ Feminino } & \multicolumn{2}{c}{ Total } \\
(anos) & $\mathbf{N}$ & $\%$ & $\mathbf{N}$ & $\%$ & $\mathbf{N}$ & $\%$ \\
\hline $\mathbf{2 0}-\mathbf{2 5}$ & 69 & 10,9 & 0 & 0,0 & 69 & 11,0 \\
$\mathbf{2 6 - 3 4}$ & 376 & 59,8 & 3 & 75,0 & 379 & 60,0 \\
$\geq \mathbf{3 5}$ & 183 & 29,1 & 1 & 25,0 & 184 & 29,1 \\
Total & 628 & 100 & 4 & 100 & 632 & 100 \\
\hline
\end{tabular}

Na tabela 2 são apresentados os resultados obtidos referentes ao número de exames toxicológicos que deram positivo para uma ou mais drogas testadas por ano. Ao todo, no período estudado de 2016 a 2021, 28 amostras foram encontradas positivas (4,4\%) para uma ou mais drogas. Com relação ao número de exames realizados por ano, em 2016, foram realizados 27 exames (4,3\%); em 2017, 144 exames (22,8\%); em 2018, 299 exames (47,3\%); em 2019, 149 exames (23,6\%); em 2020, 10 exames (1,6\%) e 3 exames (0,5\%) até a data final do estudo, em janeiro de 2021, considerando o total de amostras positivas e negativas.

Tabela 2. Frequência de resultados das 28 amostras positivas detectadas pelo teste rápido de triagem que foi utilizado para analisar amostras de urina de policiais militares do Estado de Goiás durante o período de junho de 2016 a janeiro de 2021.

\begin{tabular}{|c|c|c|c|c|c|c|c|c|}
\hline \multirow{3}{*}{$\begin{array}{l}\text { Drogas } \\
\text { Psicoativas } \\
\text { Positivas }\end{array}$} & \multicolumn{6}{|c|}{ Ano } & & \\
\hline & \multicolumn{2}{|c|}{2016} & \multicolumn{2}{|c|}{2017} & \multicolumn{2}{|c|}{2018} & \multicolumn{2}{|c|}{ Total } \\
\hline & $\mathbf{N}$ & $\%$ & $\mathbf{N}$ & $\%$ & $\mathbf{N}$ & $\%$ & $\mathbf{N}$ & $\%$ \\
\hline Cocaína & 5 & 35,7 & 0 & 0,0 & 0 & 0,0 & 5 & 17,9 \\
\hline Opiáceos & 0 & 0,0 & 1 & 16,6 & 2 & 25,0 & 3 & 10,8 \\
\hline Maconha & 1 & 7,1 & 1 & 16,6 & 3 & 37,5 & 5 & 17,9 \\
\hline $\begin{array}{l}\text { Antide- } \\
\text { pressivos }\end{array}$ & 0 & 0,0 & 1 & 16,6 & 0 & 0,0 & 1 & 3,6 \\
\hline $\begin{array}{l}\text { Benzodia- } \\
\text { zepínicos }\end{array}$ & 8 & 57,1 & 3 & 50,0 & 3 & 37,5 & 14 & 50,0 \\
\hline Total & 14 & 100 & 6 & 100 & 8 & 100 & 28 & 100 \\
\hline
\end{tabular}


A figura 1 evidência o número de resultados positivos, anualmente, das amostras de urinas de policiais militares do Estado de Goiás. No período analisado, observou-se resultados positivos para substâncias psicotrópicas somente nos anos de 2016, 2017 e 2018. No ano de 2016 houve um total de 14 amostras positivas, sendo esse, o ano com maior prevalência de amostras positivas; 2017 com 6 amostras positivas; e 2018 com 8 amostras positivas.

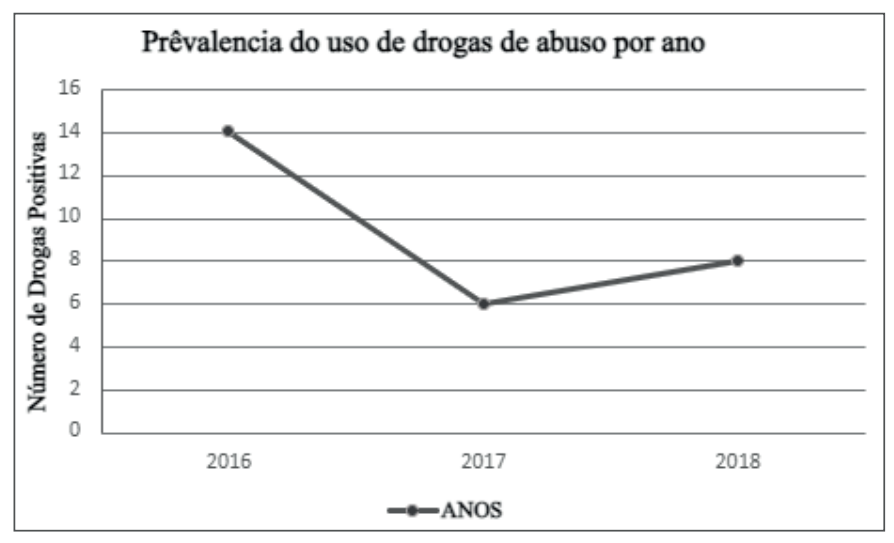

Figura 1. Frequência anual dos resultados positivos para substâncias psicotrópicas após análise com realização de teste imunocromatográfico em amostras de urinas de policiais militares no Estado de Goiás no período de junho de 2016 a janeiro de 2021.

Na figura 2 são demonstradas as cinco drogas mais comumente encontradas nas amostras de urina analisadas pelo teste imunocromatográfico MultiDrogas Rapid Test. Sendo que os benzodiazepínicos apresentaram uma prevalência de 50,0\%, cocaína $17,9 \%$, maconha $17,9 \%$, opiáceos $10,8 \%$ e antidepressivos $3,6 \%$.

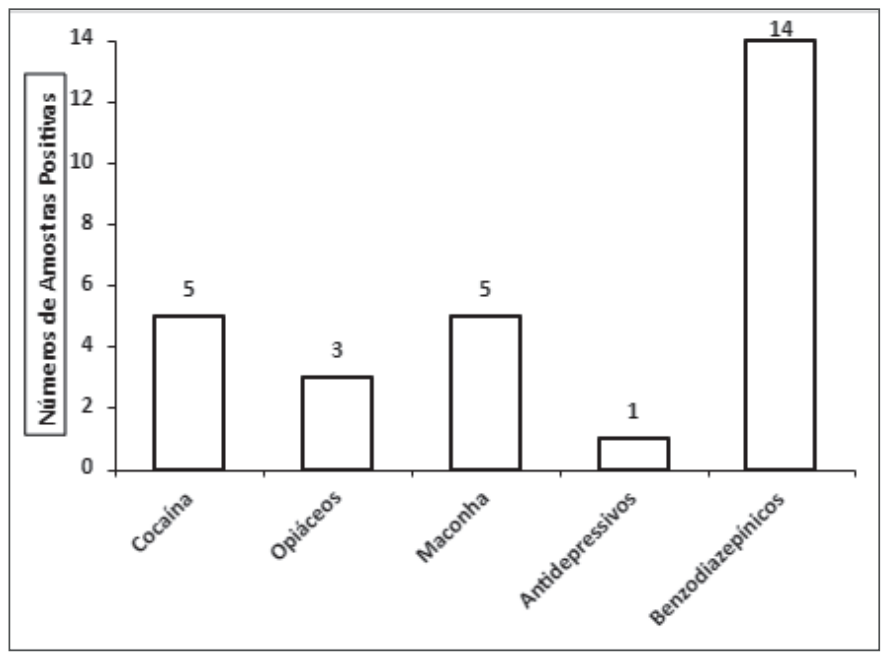

Figura 2. Drogas mais comuns encontradas nas amostras de urina de policiais militares do Estado de Goiás analisadas no período de junho de 2016 a janeiro de 2021.
Foram encontradas quatro amostras positivas para mais de uma droga, correspondendo a $14,9 \%$ do total de amostras positivas, dentre as drogas encontradas simultaneamente estão: Cocaína, Benzodiazepínicos e Maconha, todas identificadas no ano de 2016. O uso dessas drogas foi correlacionado da seguinte maneira: primeira e segunda amostra: benzodiazepínicos e cocaína. Terceira amostra: benzodiazepínicos, cocaína e maconha. Quarta e última amostra: benzodiazepínicos e cocaína.

\section{Discussão}

O presente estudo baseado em um levantamento de dados referentes ao período de junho de 2016 a janeiro de 2021, analisou a prevalência do uso de psicotrópicos por policiais militares do Estado de Goiás, atendidos no Hospital do Policial Militar (HPM), sendo obtida uma prevalência de amostras positivas de 4,4\% ( $n=28)$ no período estudado.

É importante ressaltar que, neste estudo, foram analisados dois parâmetros: gênero e idade. Como se pode ver, há uma quantidade inferior de amostras pertencentes a policiais do gênero feminino $(0,6 \%)$, quando comparada a policiais do gênero masculino $(99,4 \%)$, de um total de 632 amostras, apenas quatro amostras são do gênero feminino e, dentre estas, nenhuma foi positiva para alguma das drogas analisadas.

O possível motivo desta quantidade inferior pode estar relacionado ao número de vagas reservadas para ambos os gêneros nos editais de concurso público do Estado de Goiás para ingresso na Polícia Militar, em que apenas 10\% do total de vagas é destinada para o gênero feminino. Tendo como base os dois últimos editais, o mais antigo divulgado em 17 de outubro de 2012, com 1.050 vagas para Soldado de $2^{\circ}$ classe e, desse total, 105 (10\%) vagas eram reservadas para pessoas do gênero feminino e 945 (90\%) para o gênero masculino ${ }^{18}$. O edital mais recente e último, até a presente data, foi divulgado em 06 de setembro de 2016 visando o provimento de 2.420 vagas para o cargo de Soldado de $3^{\text {a }}$ Classe, onde apenas 242 (10\%) foram ofertadas para o gênero feminino e o restante de 2.178 para o gênero masculino $(90 \%)^{19}$.

Em outras corporações, como a Polícia Militar do Estado de São Paulo, não há essa restrição em seus editais. De acordo com os quatro últimos editais em 2017, 2018, 2019 e 2021 para provimento dos cargos de Soldado PM do Quadro de Praças de Polícia Militar (QPPM), o número total de vagas foram destinadas a candidatos do gênero masculino e feminino, sem distinção ${ }^{20-23}$. Os maiores pontuadores são classificados independentemente do gênero. Em 2013, a revista "Mulheres na Segurança Pública" destacou que, a partir de 1970, as mulheres começaram a fazer parte dessa perspectiva relativamente 
predominante do gênero masculino, ingressando no quadro de Policiais Militares do Estado de São Paulo e, a partir desse marco, foram incorporadas também nas demais unidades da federação, para compor o sistema de segurança pública ${ }^{24}$.

Um relatório de 2020 (Ano-base 2018) realizado pela Secretaria Nacional de Segurança Pública (SENASP), sobre a Pesquisa Perfil das Instituições de Segurança Pública (PISP), mostrou em um levantamento o efetivo da Polícia Militar por gênero masculino e feminino de 27 Estados do Brasil, sendo que o gênero feminino ainda é minoria. Esses autores mostram que, a maior diferença se localiza no Rio Grande do Norte, onde as mulheres representam apenas 3\% (178) do total do efetivo, em seguida está o Acre com apenas 10\% (299) do efetivo formado por pessoas do gênero feminino. Infelizmente, informações sobre o Estado de Goiás constam como dado não informado ${ }^{25}$.

Já no parâmetro idade, observou-se no presente estudo que a faixa etária predominante encontra-se entre 26 e 34 anos (59,8\%). De acordo com o II Levantamento Domiciliar realizado em 2005, evidenciou-se que 5,3\% de 1.680 entrevistados que possuem entre 25 e 34 anos já realizaram o uso de benzodiazepínicos ao longo da vida ${ }^{26}$. Outro estudo realizado no mesmo ano afirma que este uso elevado se dá devido aos distúrbios de sono e ansiedade que se tornam mais presentes nessa faixa etária, além de outros fatores relacionados ao cotidiano, que também influenciam de forma direta para este uso ${ }^{27}$.

Dentro da carreira militar isso não é diferente, é de conhecimento geral que a vida policial causa desgastes físicos e psicológicos devido aos riscos iminentes vividos diariamente durante o exercício de suas funções, o que, consequentemente, os leva a vivenciarem constantemente conflitos emocionais em crises com pouca possibilidade de controle ${ }^{28-31}$. Por lidarem com muitas adversidades, os policiais se tornam susceptíveis a altos níveis de estresse, levando-os a uma diversidade de sentimentos conflituantes, uma vez que estes devem manter o equilíbrio emocional para cumprirem suas obrigações de maneira racional visando à preservação da segurança pública e também a sua integridade física ${ }^{32-35}$.

O uso de substâncias psicoativas, acarreta a urgência de controle estrito e adequado contra as mesmas, no consumo pela classe militar que acaba levando à dependência e à destruição, tanto da classe como da humanidade no geral ${ }^{36,37}$.

Em 2013, uma pesquisa realizada em Cuiabá e Várzea Grande concluiu que 8,9\% de 237 policiais, que responderam ao questionário, já realizaram o uso de substâncias psicotrópicas na vida. O mesmo estudo afirmou que isso se dá devido ao grande desgaste causado pela profissão ${ }^{38}$. No ano de 2008, outro estudo realizado com 299 policiais do Estado de Goiás, através de um teste de triagem imunocromatográfico, apre- sentou uma frequência de $2,3 \%$ de amostras positivas, sendo os seguintes percentuais: $0,3 \%$ para anfetaminas; $0,7 \%$ para canabinoides e 1,3\% para benzodiazepinicos ${ }^{39}$.

Através da mesma metodologia, nosso estudo apresentou um dado superior ao de Nascente et al, com uma frequência de $4,4 \%$ de amostras positivas em 632 policiais analisados. Dessa forma, é possível observar que, apesar de não possuir muitos estudos sobre o presente assunto, é notória a presença do uso de substâncias psicotrópicas entre Policiais Militares e também um aumento gradativo desse uso.

De acordo com o Levantamento Domiciliar sobre o uso de drogas psicotrópicas no Brasil, realizado nos anos de 2001 e 2005, a região Centro-oeste do país apresentou um aumento de $2,7 \%$ para $3,6 \%$ de uso na vida de benzodiazepínicos ${ }^{40}$. Em 2008, o estudo anteriormente citado, realizado nas cidades de Goiânia e Aparecida de Goiânia apresentou uma prevalência de 1,3\% de amostras positivas para o uso de benzodiazepínicos por Policiais Militares ${ }^{41}$. De acordo com o exposto, esse dado é inferior ao do presente estudo, em que 2,2\% das amostras analisadas foram positivas para benzodiazepínicos por militares. Em ambos os estudos, foi possível observar que a prevalência do uso de benzodiazepínicos está em maior porcentagem de amostras positivas na vida policial com um aumento gradativo e significativo.

No presente estudo verificou-se que a droga utilizada com maior frequência foi a classe de benzodiazepínicos com uma prevalência de 2,2\%, que constitui o grupo de psicotrópicos mais comumente utilizado na prática clínica, devido as suas quatro atividades principais: ansiolítica, hipnótica, anticonvulsivante e relaxante muscular. Embora tal substância, aparentemente, seja de um grupo de substâncias que quando utilizadas de forma correta, pode ajudar bastante no tratamento psicológico, por outro lado, quando utilizadas em excesso podem provocar efeito paradoxal, devendo tal uso ser acompanhado com muita cautela, principalmente na classe militar. Essa categoria profissional, é portadora de armamento e o uso indiscriminado de drogas pode trazer situações de risco tanto para sua própria segurança, quanto para a segurança dos que estão ao redor.

\section{Conclusão}

Pode-se concluir, a partir dos resultados obtidos no presente estudo, que foi possível delimitar a prevalência do uso de drogas de abuso em policiais militares, integrantes do quadro de segurança pública do Estado de Goiás. A partir do levantamento realizado, pode-se constatar que a maioria dos policiais participantes foi do gênero masculino Dentre as 28 amostras positivas encontradas, a prevalência de uso mais 
comum foi para benzodiazepínicos. Considerando dessa forma ser possível correlacionar os diversos fatores estressantes da rotina desses profissionais com o uso elevado dessa classe de medicamentos.

Não obstante, podemos perceber que não existe uma quantidade significativa de estudos sobre o uso de drogas de abuso por policiais militares no Estado de Goiás, contudo, não deixa de ser uma temática importante. Enfatizamos que seria de grande valia a implantação de exames para a detecção do uso de drogas no programa já existente do centro de saúde integral do policial militar (CSIPM), avaliando, anualmente, o estado clínico desses servidores, zelando pela saúde clínica, física e psicológica e com o intuito de ajudar a minimizar as práticas de tal uso.

\section{Referências}

1. Martello S, Fett CA. Uso de drogas psicotrópicas por Policiais Militares de Cuiabá e Várzea Grande. Homens Do Mato - Revista Científica de Pesquisa em Segurança Pública. 2013;11:1-20.

2. Organización Mundial de la Salud. Guia de la buena prescripcion. Genebra: OMS, 1994.

3. Martello S, Fett CA. Uso de drogas psicotrópicas por Policiais Militares de Cuiabá e Várzea Grande. Homens Do Mato - Revista Científica de Pesquisa em Segurança Pública. 2013;11:1-20.

4. Organização Mundial de Saúde. Relatório sobre A saúde no mundo 2001: saúde mental: nova concepção, nova esperança. Genebra: 2001. 135p

5. Centro Brasileiro de Informação de Medicamentos Psicotrópicos (Cebrim) - Universidade Federal de São Paulo (Unifesp). Departamento de Psicofarmacologia. Haja ansiedade. Haja insônia. Bol. CEBRID, N. 47, Jan./ Mar. 2003

6. Lucas ACS, Parente RCP, Picanço NS, Conceição DA, Costa KRC, Magalhães IRS, Siqueira JCA. Uso De Psicotrópicos entre Universitários da Área de saúde da Universidade Federal do Amazonas, Brasil. Cad Saude Publica 2006; 22(3):663-671.

7. Escritório das Nações Unidas sobre drogas e Crime (UNODC). Relatório Mundial sobre Drogas. Eua: Unodc; 2012.

8. United Nations Office On Drugs And Crime (UNODOC). Relatório Mundial Sobre Drogas 2020: Consumo Global De Drogas Aumenta, Enquanto COVID-19 Impacta Mercados, Aponta Relatório [Internet]. Viena, 25 De Junho De 2020 [atualizado em 2020 jun. 25; citado em 2021 out. 19]. Disponível em: www.unodc.org.

9. Bastos FIPM, et al. (Org.). III Levantamento Nacional
Sobre O Uso De Drogas Pela População Brasileira. Rio De Janeiro: FIOCRUZ/ICICT, 2017. 528.

10. Andrade LP, Costa SL, Marquetti FC. A Rua Tem Um Imã, Acho Que É Liberdade: Potência, Sofrimento E Estratégias De Vida Entre Moradores De Rua Na Cidade De Santos, No Litoral Do Estado De São Paulo. Saúde Soc. 2014 Oct; 23:1248-1261.

11. Rodriguez VMR, Scherer ZAP. Motivação Do Estudante Universitário Para O Consumo De Drogas Legais. Latino-am 2008 Jun;16(spe): 572.

12. Fernandes MA, Silva JSE, Vilarinho JOV, Seabra LO, Feitosa CDA. Uso de substâncias psicoativas por profissionais de saúde: revisão integrativa. SMAD Revista Eletrônica Saúde Mental Álcool E Drogas. 2018;13(4): 221-231.

13. Vieira GCG, Brida RL, Macuch RS, Massuda EM, Preza GP. Uso De Psicotrópicos Pelo Enfermeiro: Sua Relação Com O Trabalho. Cinergis. 2016 Set; 17(3): 191-195

14. Bahora M, Sterk CE, Elifson KW. Understanding Recreational Ecstasy Use In The United States: A Qualitative Inquiry. Int J Drug Policy 2009 Jan; 20(1): 62-69.

15. Verstraete AG, Pierce A. Workplace Drug Testing In Europe. Forensic Sci Int 2001 Set;121(1-2): 2-6.

16. Meririnne $E$, Mykkänen $S$, Lillsunde $P$, Kuoppasalmi K, Lerssi R, Laaksonen I, Lehtomäki K, Henriksson M.Workplace Drug Testing In A Military Organization: Results And Experiences From The Testing Program In The Finnish Defence Forces. Forensic Sci Int 2007;170(2-3): 171-174.

17. Cruz MS, Impacto Do Uso De Drogas Na População Brasileira: Análise De Dados Epidemiológicos De Indicadores - 2001 A 2007. In: Duarte PCAV, Stempliuk VA, Barroso LP, Organizadoras. Relatório Brasileiro Sobre Drogas; SENAD 2009: 345-357.

18. Edital Concurso Polícia Militar Goiás 2012 [Internet]. [Cited 2021 Oct 19]. Disponível em: Https://Www. Acheconcursos.Com.Br/Edital-concurso/Edital-concurso-policia-militar-goias-2012

19. Edital Concurso PM-GO 2016 [Internet]. www. Acheconcursos.Com.Br. [Citado 2021 Out 19]. Disponível Em: Https://Www.Acheconcursos.Com.Br/ Edital-concurso/Edital-concurso-pm-go-2016

20. Edital Concursos PM-SP 2017 - Soldado [Internet]. [Citado 2021 Out 19]. Disponível Em: https://Www. Acheconcursos.Com.Br/Edital-concurso/Edital-concurso-pm-sp-2017-soldado

21. Edital Concursos PM-SP 2018 [Internet]. [Citado 2021 Out 19]. Disponível Em: Https://Www.Acheconcursos. 
Com.Br/Edital-concurso/Edital-concurso-pm-sp-2018

22. Secretaria Da Segurança Pública Polícia Militar Do Estado De São Paulo. Disponível em: Https://Documento. Vunesp.Com.Br/Documento/Stream/Otcwotgw

23. Edital Concurso PMSP 2021 - Soldados [Internet]. [Cited 2021 Oct 19]. Available From: Https://www. Acheconcursos.Com.Br/Edital-concurso/Edital-pmsp2021-soldado

24. Brasil. Secretaria Nacional De Segurança Pública. (Senasp). Mulheres Nas Instituições De Segurança Pública: Estudo Técnico Nacional. Brasília, 2013. P. 10

25. Araujo JC, Sousa RR (Coord.). Coleção Pesquisa perfil das instituições de segurança pública - Ano-base 2018. Edição digital ministério da justiça e segurança pública/secretaria nacional de segurança pública 2020.

26. Carlini EA. (Super.) II Levantamento Domiciliar sobre o uso de drogas psicotrópicas no Brasil: Estudo envolvendo as 108 maiores cidades do País: 2005, São Paulo (sp): Senad; 2007.

27. Orlandi P, Noto AR. Uso indevido de benzodiazepínicos: um estudo com informantes-chave no município de São Paulo. Revista Latino-Americana de Enfermagem 2005; 13: 896-902.

28. Costa SHN et al. Survey on the use of psychotropic drugs by twelve military police units in the municipalities of Goiânia and Aparecida de Goiânia, state of Goiás, Brazil. Brazilian Journal of Psychiatry 2010; 32 (4): 389-395.

29. Bray RM, Hourani LL. Substance use trends among active duty military personnel: findings from the united states department of defense health related behavior surveys, 1980-2005 2007 Jul; 102 (7): 1092-101.

30. Maia DB et al. Post-traumatic stress symptoms in an elite unit of brazilian police officers: prevalence and impact on psychosocial functioning and on physical and mental health. J affect disord 2007; 97: 241-5.

31. Viana N. Inspeção do trabalho E violência nas relações de trabalho. In: José Fernando da Silva; Ricardo Barbosa Lima; Sadi Dal Rosso. (Org.). Violência e trabalho no brasil. Cegraf, 2001; 1:133-153.

32. Maia DB et al. Post-traumatic stress symptoms in an elite unit of brazilian police officers: prevalence and impact on psychosocial functioning and on physical and mental health. J affect disord 2007; 97: 241-5.

33. Amador FS. Violência Policial: Verso e reverso do sofrimento. Dissertação de mestrado. Instituto de psicologia, pontifícia universidade católica do Rio Grande do Sul, Porto Alegre, 1999.
34. Gomes R, Minayo MCS, Silva CFR. Riscos da profissão. In: Minayo, M. C. S.; Souza, E. R. (Orgs). Missão investigar: Entre o ideal e a realidade de ser policial. Rio de Janeiro: Garamond, 2003.

35. Patrocínio MCC, Souza EMCD. Fatores psicossociais na gênese de atos agressivos de policiais militares, in: Sampaio, J. R. (Org.). Qualidade de vida no trabalho e psicologia social. 2.Ed. São Paulo: Casa do psicólogo, 2004.

36. Verstraete $A G$, Pierce A. Workplace drug testing in europe. Forensic sci int 2001; 121(1-2):2-6.

37. Meririnne $E$, Mykkänen S, Lillsunde P, Kuoppasalmi K, Lerssi R, Laaksonen I, Lehtomäki K, Henriksson M. Workplace drug testing in a military organization: results and experiences from the testing program in the finnish defence forces. Forensic sci int 2007; 170(2- 3):171-174.

38. Martello S, Fett CA. Uso de drogas psicotrópicas por Policiais Militares de Cuiabá e Várzea Grande. Homens Do Mato - Revista Científica de Pesquisa em Segurança Pública. 2013 Jul/Dez; 11; 1-20.

39. Costa SHN et al. Prevalência do uso de drogas psicotrópicas em unidades da Polícia Militar. Ciência \& Saúde Coletiva. 2015, V. 20, N. 6, pp. 1843-1849.

40. Carlini, EA. (Super.) et al. II Levantamento Domiciliar sobre o uso de drogas psicotrópicas no Brasil: Estudo envolvendo as 108 maiores cidades do País: 2005, São Paulo (sp): Senad; 2007.

41. Costa SHN et al. Prevalência do uso de drogas psicotrópicas em unidades da Polícia Militar. Ciência \& Saúde Coletiva 2015; 20 (6): 1843-1849. 